\title{
Elemental Fingerprint of Herbal Medicines Formed by Inductively Coupled Plasma Atomic Emission Spectroscopy (ICP-AES)
}

\author{
Yongjun Zheng ${ }^{1, *}$, Kang Zheng ${ }^{2}$, Erdong Liu ${ }^{1}$ and Yantuan $\mathrm{Li}^{2}$ \\ ${ }^{1}$ College of Chemistry and Chemical Engineering, Qufu Normal University, Qufu, Shandong,273165, P.R. China \\ 2 Marine Drug \& Food Institute, Ocean University of China, Qingdao, 266003, P.R. China
}

Received 15 May 2013; Accepted 25 July 2013

\begin{abstract}
Herbal medicines contain multiple compounds, each of which may be relevant to the medicine's pharmacological activity. A method referred to as 'elemental fingerprint', which is accurate and rapid, is used in this paper. It provides a more powerful approach than the single component analysis to the quality control of herbal medicines as it furnishes information on several elements and the ratios at which they are present. Eight elements, $\mathrm{Li}, \mathrm{Mn}, \mathrm{Ni}, \mathrm{Zn}, \mathrm{Cd}, \mathrm{Pb}, \mathrm{Se}, \mathrm{Cu}$, in 21 samples of herbal medicines were measured by inductively coupled plasma atomic emission spectroscopy (ICPAES). The data analysis was performed with EXCEL and SPSS. The experimental results clearly demonstrated that the proposed method was a useful tool to control the quality of herbal medicines.
\end{abstract}

Keywords: Herbal Medicines, Elemental Fingerprint, ICP-AES

\section{Introduction}

Herbal medicines, which have been in the Chinese material medicines and other folk medicines for thousands of years, are being increasingly used nowadays in the whole world for improving health conditions of humans as well as for the prevention and treatment of various diseases [1]. However, as herbal medicines may contain hundreds of ingredients, our knowledge about their pharmacological activities is still limited [1]. Furthermore, the analytical methods for the quality control of herbal medicines have thus far not been standardized [2],[3]. Traditionally, according to their different morphology, the identification of herbal medicines is carried out by the experienced herbalist doctor, which prevents the spread of herbal medicines [4]. The key factor is the construction of both quality control standards for raw materials and the standardization of finished herbal drugs. Up to date, variation methods had been designed to solve aforementioned problems. For example, chromatographic fingerprint analysis, which uses modern chromatographic techniques such as gas chromatograph (GC), highperformance liquid chromatography (HPLC) or highperformance thin layer chromatography (HPTLC) to construct specific patterns of recognition for multiple compounds in herbal medicine, has been adopted to identify the quality of herbal medicines [5]. However, the current spectroscopic fingerprinting technology that aimed at specific organic components, not suitable for the complex multi-component environment. Furthermore, elemental fingerprint techniques, based on elemental composition and

\footnotetext{
*E-mail address:zyj6787@163.com
}

ISSN: 1791-2377@ 2013 Kavala Institute of Technology. All rights reserved. multivariate statistical analysis, have been used to solve this problem [5] [6]. elemental fingerprint techniques.

In this article, we further developed elemental fingerprint techniques to control the quality of herbal medicines. The contents of $\mathrm{Li}, \mathrm{Mn}, \mathrm{Ni}, \mathrm{Zn}, \mathrm{Cd}, \mathrm{Pb}, \mathrm{Se}$, and $\mathrm{Cu}$ in 21 species of herbal medicines have been determined by using inductively coupled plasma atomic emission spectrometry (ICP-AES) to establish the elemental fingerprint.

\section{Experimental}

\subsection{Instrument}

An Optima 4300DV plasma emission spectrometer (Perkin Elmer Corporation, United States) was used for the determination of analytes.MDS-10 microwave digestion system (Sineo Microwave Chemistry Technology (China) Co., LTD) was used to prepare the samples.

\subsection{Reagents and materials}

All reagents were of analytical-reagent grade, and distilled, deionized water was used throughout. Standard Solution: Using the standard solution provided by Perkin Elmer. We prepared the mixed standard solution according to the concentration range of each element in samples.

\subsection{Preparation of samples}

Samples of 21 herbal medicines that were most frequently consumed were obtained commercially from a local store of Chinese traditional medicines in Qingdao, Shandong Province, China. Each of the samples had been identified by an experienced herbalist. All the samples from the packages 
were ground into a fine powder and dried at $80{ }^{\circ} \mathrm{C}$ for $24 \mathrm{~h}$ prior to use.

$0.5000 \mathrm{~g}$ sample that was accurately weighed was put into Teflon digestion tank; added $5 \mathrm{~mL}$ concentrated $\mathrm{HNO}_{3}$ soaking overnight. Then we added $3 \mathrm{~mL} \mathrm{H}_{2} \mathrm{O}_{2}$, digested in the microwave digestion system for 8 min until completely, transferred to $50 \mathrm{~mL}$ volumetric flask diluted using $5 \%$ $\mathrm{HNO}_{3}$ solution to the mark. Two parallel copies of the blank were prepared in the same way.

\subsection{Determination by ICP-AES method}

Booting the system into Winlab 32 operating software by Optima 4300DV plasma emission spectrometer operating procedures, establishing the analytical methods, Plasma ignited. The blank solution, standard solution and sample solution was determined in order. Instrument operating parameters are listed in Table 1 and Table 2.

\begin{tabular}{cc} 
Table 1. Operation Parameters of Optima 4300DV & \\
\hline Name & Parameters \\
\hline Nebulizer pressure $(\mathrm{KPa})$ & 125.0 \\
Cooling gas flow rate $(\mathrm{L} / \mathrm{min})$ & 0.8 \\
Carrier gas flow rate $(\mathrm{L} / \mathrm{min})$ & 15 \\
Auxiliary gas flow rate $(\mathrm{L} / \mathrm{min})$ & 0.2 \\
Peristaltic pump flow rate $(\mathrm{mL} / \mathrm{min})$ & 1.5 \\
Nebulizer flow rate $(\mathrm{L} / \mathrm{min})$ & 0.8 \\
\hline
\end{tabular}

\begin{tabular}{cccc}
\multicolumn{5}{l}{ Table 2. Detection wavelengths(nm-1) } \\
\hline $\mathrm{Li}$ & 670.784 & $\mathrm{Mn}$ & 257.610 \\
\hline $\mathrm{Cd}$ & 228.802 & $\mathrm{Ni}$ & 231.604 \\
$\mathrm{Cu}$ & 327.393 & $\mathrm{~Pb}$ & 220.353 \\
$\mathrm{Zn}$ & 206.200 & $\mathrm{Se}$ & 196.026 \\
\hline
\end{tabular}

\subsection{Data analysis}

For the chemometric study, each of the herbal medicines samples was characterized by 8 chemical descriptors which are the contents of the analyzed elements. The software EXCEL2003 (Microsoft Corporation, USA) was applied to plot a bar graph from the data matrix composed of 8 columns (the analyzed elements) and 21 rows (herbal medicines samples). The statistical package SPSS 17.0 (SPSS Inc., USA) was used for statistical analysis.

\section{Results and discussion \\ 3.1 Elements in herbal medicines}

Herbal medicines contain various elements. Some of these elements are benefit to human beings such as $\mathrm{Mn}, \mathrm{Cu}, \mathrm{Zn}$, $\mathrm{Se}$, etc., while others are non-essential to humans or even toxic elements such as $\mathrm{Cd}, \mathrm{Pb}$ [7], [8]. Much research have been made to establish the normal concentration range and evaluate their role in herbal medicines [9], [10]. To construct fingerprints of herbal medicines, eight elements in 21 samples are determined. The mean concentrations of elements determined in this study are shown in Table 3. As can be seen from these values, $\mathrm{Mn}$ and $\mathrm{Zn}$ are the elements with a major content in all samples, with average concentrations of 44.6501 and $43.7744 \mu \mathrm{g} / \mathrm{g}$, respectively. While other elements present lower contents, their average values are less than $5 \mu \mathrm{g} / \mathrm{g}$. Several trace elements, such as $\mathrm{Mn}, \mathrm{Zn}, \mathrm{Cu}$ and $\mathrm{Se}$, are chemical elements that play an important role as oligo elements in biological systems. However, these micronutrient elements may also be the origin of adverse effects on living organisms if the dosages exceed certain levels.

Table 3. Contents of elements in the herbal medicines samples, presented as means $(n=6)$

\begin{tabular}{|c|c|c|c|c|c|c|c|}
\hline $\begin{array}{c}\text { Element } \\
(\mu \mathrm{g} / \mathrm{g})\end{array}$ & Baixianpi & Baizhi & Baihe & Dingxiang & Honghua & Huluba & Jiujie changpu \\
\hline $\mathrm{Li}$ & 0.3688 & 0.6000 & 0.4153 & 0.1152 & 1.6580 & 0.8201 & 0.4124 \\
\hline $\mathrm{Mn}$ & 9.9083 & 15.3958 & 15.7309 & 248.6279 & 24.8103 & 20.1700 & 28.2400 \\
\hline $\mathrm{Ni}$ & 1.9837 & 3.1374 & 3.1923 & 1.9944 & 4.0286 & 2.2450 & 0.4359 \\
\hline $\mathrm{Zn}$ & 11.9617 & 33.9332 & 38.2620 & 25.6481 & 50.5094 & 55.8300 & 46.7200 \\
\hline $\mathrm{Cd}$ & 1.5849 & 1.9646 & 2.6418 & 1.5765 & 1.6580 & 0.0968 & 0.1504 \\
\hline $\mathrm{Pb}$ & 0.2871 & 0.3942 & 1.4881 & 8.6217 & 6.9414 & 1.5800 & 4.8710 \\
\hline $\mathrm{Se}$ & 1.5230 & 0.2320 & 1.8063 & 1.4580 & 0.6694 & 3.3540 & 3.0160 \\
\hline $\mathrm{Cu}$ & 3.7842 & 1.5109 & 4.2880 & 4.3799 & 9.6153 & 0.6055 & 7.6514 \\
\hline $\begin{array}{c}\text { Element } \\
(\mu \mathrm{g} / \mathrm{g})\end{array}$ & Huanglian & Juemingzi & Lianzi & Ruxiang & Hujisheng & Tianma & Xiaohuixiang \\
\hline $\mathrm{Li}$ & 0.3158 & 0.1732 & 0.0098 & 0.1613 & 0.3558 & 0.1583 & 4.1400 \\
\hline $\mathrm{Mn}$ & 117.9000 & 10.3627 & 44.1600 & 19.5000 & 46.5200 & 9.7842 & 24.2300 \\
\hline $\mathrm{Ni}$ & 2.9260 & 2.5160 & 2.6360 & 0.4132 & 0.4245 & 2.1792 & 1.2900 \\
\hline $\mathrm{Zn}$ & 156.3000 & 69.8100 & 37.4900 & 6.5741 & 9.1482 & 28.1913 & 39.7500 \\
\hline $\mathrm{Cd}$ & 2.1440 & 1.4400 & 1.4851 & 0.1815 & 0.4503 & 1.8693 & 0.0000 \\
\hline $\mathrm{Pb}$ & 10.4200 & 0.7813 & 0.3178 & 2.3660 & 3.6470 & 0.9194 & 3.0300 \\
\hline $\mathrm{Se}$ & 2.6650 & 2.3630 & 0.4900 & 6.3660 & 2.8100 & 6.4599 & 0.6200 \\
\hline $\mathrm{Cu}$ & 12.6100 & 9.3090 & 11.7740 & 2.3795 & 5.4179 & 0.6812 & 0.5711 \\
\hline $\begin{array}{c}\text { Element } \\
(\mu \mathrm{g} / \mathrm{g})\end{array}$ & Yinchen & Yinxingye & Yuzhu & Pangdahai & Zisuzi & Jinqiancao & Ziyulan \\
\hline $\mathrm{Li}$ & 2.7090 & 3.0450 & 0.7053 & 0.0299 & 0.9882 & 2.6040 & 0.0499 \\
\hline $\mathrm{Mn}$ & 63.1400 & 13.6800 & 32.2174 & 22.2554 & 36.5396 & 99.0296 & 35.4500 \\
\hline $\mathrm{Ni}$ & 2.7560 & 2.3120 & 2.4538 & 1.6267 & 0.9882 & 2.0510 & 5.1210 \\
\hline $\mathrm{Zn}$ & 25.4600 & 21.3200 & 26.8528 & 42.8443 & 30.2475 & 0.0000 & 162.4100 \\
\hline $\mathrm{Cd}$ & 0.2360 & 1.5770 & 2.2253 & 1.6766 & 0.0540 & 0.9496 & 1.8966 \\
\hline $\mathrm{Pb}$ & 4.7820 & 3.5200 & 1.8478 & 0.8583 & 1.6324 & 11.7172 & 0.0000 \\
\hline $\mathrm{Se}$ & 0.5940 & 0.0000 & 0.6656 & 0.0000 & 2.0017 & 0.8671 & 2.6951 \\
\hline $\mathrm{Cu}$ & 16.6200 & 4.5547 & 0.7513 & 0.8064 & 3.8754 & 7.5537 & 7.4266 \\
\hline
\end{tabular}




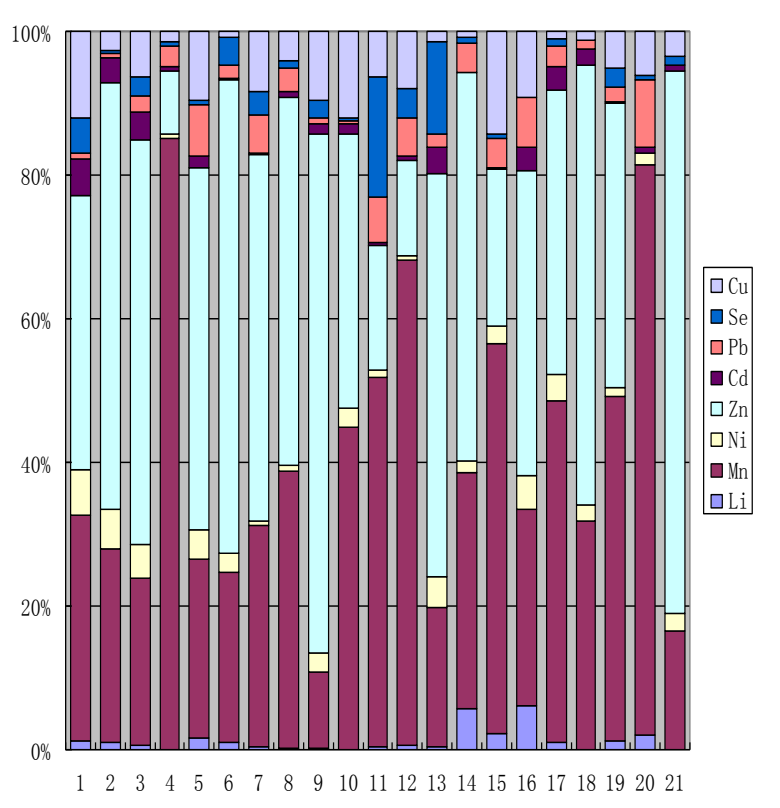

Fig 1. Elemental fingerprints of 21 herbal medicines samples.

$1=$ Baixianpi; 2 = Baizhi;3 = Baihe; 4 = Dingxiang; $5=$ Honghua; 6 = Huluba; 7 = Jiujie changpu; $8=$ Huanglian; 9 = Juemingzi; 10 = Lianzi; 11 = Ruxiang; 12 = Hujisheng; $13=$ Tianma; $14=$ Xiaohuixiang; $15=$ Yinchen; $16=$ Yinxingye; $17=$ Yuzhu; $18=$ Pangdahai; $19=$ Zisuzi; $20=$ Jinqiancao; 21 = Ziyulan.

\subsection{Fingerprint analysis of herbal medicines}

As mentioned above, the analysis of one or more compounds as markers fails to identify a specific herbal medicine, because the components or markers are not unique to a specific herbal medicines and an active ingredient of a certain herbal medicine can be found in many other herbs [9] The fingerprint of herbal medicines is an identity of single herbal medicine via building a unique feature. In fact, different kind of features can be selected to represent the herbal medicines and can be used as fingerprints. In the present paper, the mean contents of eight elements have been determined in samples of herbal medicines to establish elemental fingerprint for quality control by using ICP-AES. For convenience of recognition, the data obtained from the experiment are transformed to plot a percentage-stacked graph (Fig. 1). Because the concentration and proportion of elements are different among these herbal medicines, it can be easily found that each herbal medicine is corresponding with a unique fingerprint pattern. With the help of fingerprint, we can easily evaluate the identity and quality of herbal medicines.

\subsection{Evaluation of elemental fingerprint by similarity analysis}

Similarity analysis is a powerful tool in establishing and evaluating the fingerprints of herbal medicines. By using similarity analysis, the similarity of herbal medicines can be evaluated by Euclidean distance $\left(d_{r}\right)$, cosine similarity
${ }_{(} C_{r}$, and Pearson product-moment correlation coefficient $\left(r_{r}\right)$ [11]. The formulas are as followed:

$$
\begin{aligned}
& d_{r}=\left[\sum_{i=1}^{m}\left(X_{i}-Y_{i}\right)^{2}\right]^{\frac{1}{2}} \\
& C_{r}=\frac{\sum_{i=1}^{m} X_{i} \bullet Y_{i}}{\sqrt{\left(\sum_{i=1}^{m} X^{2}{ }_{i}\right)\left(\sum_{i=1}^{m} Y^{2}{ }_{i}\right)}} \\
& r_{r}=\frac{\sqrt{\sum_{i=1}^{m}\left(X_{i}-\bar{X}\right)\left(Y_{i}-\bar{Y}\right)}}{\sqrt{\sum_{i=1}^{m}\left(X_{i}-\bar{X}\right)^{2} \sum_{i=1}^{m}\left(Y_{i}-\bar{Y}\right)^{2}}}
\end{aligned}
$$

where $X_{i}$ and $Y_{i}$ are ith elements in two fingerprints and $\mathrm{m}$ is the number of element, $\bar{X}$ and $\bar{Y}$ are the average values of elements in fingerprints.

In our study, three samples of Zisuzi were determined to evaluate the fingerprint's discriminatory power. The concentrations of elements in three samples are listed in Table 4. By using SPSS, Euclidean distance, cosine similarity, and Pearson product-moment correlation coefficient can be calculated easily. Three samples shows a high degree of similarity with Zisuzi's fingerprint, giving the values of $0.846,1.169$, and 1.467 in Euclidean distance, $1.000,1.000$, and 1.000 in cosine similarity, 1.000, 1.000, and 1.000 in Pearson product-moment correlation coefficient.

Table 4. Contents of elements in the three samples of Zisuzi, presented as means $(\mathrm{n}=6)$

\begin{tabular}{cccc}
\hline $\begin{array}{c}\text { Element } \\
(\boldsymbol{\mu g} / \mathbf{g})\end{array}$ & Sample 1 & Sample 2 & Sample 3 \\
\hline $\mathrm{Li}$ & 0.9756 & 0.9868 & 0.9841 \\
$\mathrm{Mn}$ & 36.1753 & 37.6829 & 38.0057 \\
$\mathrm{Ni}$ & 0.9846 & 0.9753 & 0.9873 \\
$\mathrm{Zn}$ & 31.0025 & 30.1739 & 30.2753 \\
$\mathrm{Cd}$ & 0.0526 & 0.0530 & 0.0547 \\
$\mathrm{~Pb}$ & 1.6078 & 1.6322 & 1.6310 \\
$\mathrm{Se}$ & 2.1081 & 2.1753 & 2.0173 \\
$\mathrm{Cu}$ & 3.9021 & 3.7215 & 3.9052 \\
\hline
\end{tabular}

In brief, it can be seen that the herbal medicines can be effectively discriminated according to their concentration and proportion of elements. Each herbal medicine presented a distinctive elemental fingerprint, as was indicated by similarity analysis.

\section{Conclusion}

In this study, we developed elemental fingerprint to identify the quality of herbal medicines instead of reported 
chromatographic fingerprint. The results indicated that elemental fingerprint is an effective and reliable identity for the purpose of species authentication and quality control of herbal medicines. In addition, this method can be extended to the other herbal medicines, which may have wide potential applications in the identification of herbal medicines.

\section{Acknowledgments}

The authors are extremely grateful to the financial support from the National Natural Science Foundation of China (No. 21071133) and the Department of Education of Shandong Province, China (No.J11LB07).

\section{References}

1. Raffaele Capasso, Angelo A. Izzo, Luisa Pinto,Teresa Bifulco, Carmen Vitobello, Nicola Mascolo. "Phytotherapy and quality of herbal medicines", Fitoterapia 71, 2000, pp.58-65.

2. Valentina Razmovski-Naumovski, Wannit Tongkao-on, Benjamin Kimble, et al. "Multiple Chromatographic and Chemometric Methods for Quality Standardisation of Chinese Herbal Medicines", Mode Tradit Chin Med Mater Med 12(1), 2010, pp.99-106.

3. Andrew Flower, George Lewith, Paul Little. "Combining rigour with relevance: A novel methodology for testing Chinese herbal medicine", Journal of Ethnopharmacology 134, 2011, pp.373-378.

4. Yang Meihua, Wang Linan. "Advances in Techniques on Analysis and Removal of Pesticide Residues in Traditional Chinese Herbal Medicines", Mode Tradit Chin Med Mater Med 10(1), 2008, pp.107-112.

5. Shanshan YE, Jian YANG, Hongbo LIU and Yuji OSHIMA. "Use of Elemental Fingerprint Analysis to Identify Localities of Collection for the Large Icefish Protosalanx chinensis in Taihu Lake, China”, J. Fac. Agr., Kyushu Univ. 56 (1), 2011, pp.41-45.
6. A. Gonzálvez, A. Llorens, M.L. Cervera, S. Armenta, M. de la Guardia. "Elemental fingerprint of wines from the protected designation of origin Valencia", Food Chemistry 112, 2009, pp.2634.

7. Ernst E., "Toxic heavy metals and undeclared drugs in Asian herbal medicines", Trends Pharmacol Sci 23, 2002, pp.136-139.

8. WHO., "General Guidelines for Methodologies on Research and Evaluation of Traditional Medicines", 2000, pp.11-12.

9. Chow Pyt, Chuath, Tankf, et al. "Dilute acid digestion procedure for the determination of Lead, copper, mercury in traditional Chinese medicines by AAS", Analyst 120, 1995, pp.1221-1223.

10. Wang $X$, Zhuang $Z$, Yang $P$, et al., "Hyphented techniques combined with Atomic spectrometry for environmental studies", Microchim J. 51(1-2), 1995, pp.88-98.

11. B.S. Everitt, S. Landau, M. Leese, Cluster Analysis, fourth ed., Arnold, London, 2001. pp.56-57. 